\title{
Nuclear Magnetic Resonance Study of Nanoscale Ionic Materials
}

\author{
Joanna Mary Oommen, ${ }^{\text {a,z }}$ Muhammad Mustafa Hussain, ${ }^{a, z}$ \\ Abdul-Hamid M. Emwas, ${ }^{\text {b Praveen Agarwal, }{ }^{c} \text { and Lynden A. Archer }}{ }^{\mathrm{c}}$ \\ ${ }^{a}$ Integrated Nanotechnology Fab and ${ }^{b}$ Nuclear Magnetic Resonance Laboratory, King Abdullah University \\ of Science and Technology, Thuwal 23955-6900, Kingdom of Saudi Arabia \\ ${ }^{c}$ School of Chemical and Biomolecular Engineering, Cornell University, Ithaca, \\ New York 14853-5201, USA
}

\begin{abstract}
Nanoscale ionic materials (NIMs) are a new class of nanomaterials that exhibit interesting properties including negligible vapor pressures and tunable physical states, among others. In this study, we analyzed the temperature-wise performance of NIMs using nuclear magnetic resonance (NMR) spectroscopy. NIMs are relatively stable over a temperature range from 300 to 383 K, rendering them usable in high temperature applications. We confirmed the presence of covalent bonds between the $\mathrm{SiO}_{2}$ core and the sulfonate group and determined relative concentrations of aromatic and aliphatic hydrocarbons. These findings serve as first hand proof-of-concept for the usefulness of NMR analyses in further studies on the diffusive properties of NIMs. (C) 2010 The Electrochemical Society. [DOI: 10.1149/1.3477935] All rights reserved.
\end{abstract}

Manuscript submitted May 30, 2010; revised manuscript received July 15, 2010. Published August 13, 2010.

A new class of nanomaterials termed nanoscale ionic materials (NIMs) is attracting widespread research interest. ${ }^{1-7}$ These materials consist of an inorganic nanoparticle core with oligomeric chains densely grafted to the nanoparticles by ionic linkage (inset of Fig. 1). The unique chemical structure of NIMs allows them to overcome the problem of nanoparticle aggregation, and NIMs exhibit fluidlike behaviors in absence of any solvent. ${ }^{1-7}$ NIMs exhibit interesting properties that make them suitable for use in several applications of current relevance, e.g., carbon capture, ${ }^{8}$ photovoltaics and energy storage, ${ }^{9}$ enhanced oil recovery, and water desalination.

The characterization of the chemical structure of NIMs is essential for understanding the true nature of the chemical bonding and improving the synthesis methodologies for the various NIM applications outlined above. Several characterization experiments have been conducted to date. ${ }^{1-7}$ These studies have given a better understanding of the structure and dynamics of NIMs. In addition, several factors have impact on the fluidity of the NIM system, including the anion being attached through ionic bonds to the corona, the packing fraction of the core, and temperature conditions. ${ }^{2}$ In this study, we show the performance of NIMs in relation to temperature using NMR spectroscopy. NIMs are relatively stable over a temperature range from 300 to $383 \mathrm{~K}$, rendering them usable in high temperature applications. We also confirmed the presence of covalent bonds between the $\mathrm{SiO}_{2}$ core and the sulfonate group and correctly detected aromatic and aliphatic hydrocarbons along with their relative concentrations. These findings serve as first hand proof-of-concept for the usefulness of NMR spectroscopy in further studies on the diffusive properties of NIMs.

Magic-angle-spinning (MAS) NMR spectroscopy is an important technique in determining the structure and composition of solid materials. Cross-polarization (CP) under MAS can be used to enhance the NMR signal of nuclei with low natural abundance and low gyromagnetic ratios, such as ${ }^{13} \mathrm{C}$, by magnetization transfer from nuclei with high natural abundance and high gyromagnetic ratios, such as ${ }^{1} \mathrm{H}$. Hence, CP MAS NMR is the most appropriate technique for recording carbon spectra of polymers and polymer composites with solid-state NMR. Additionally, the high specificity of NMR enables an accurate estimation of the amount of any element with an active isotope and correlates bonded elements. Finally, solid-state NMR also offers a temperature variation feature to study the type of bonding and other temperature effects.

To our best knowledge, this was the first time that an NMR study has been carried out on NIMs. Hence, the primary focus was on confirming the effectiveness of NMR on NIM samples in addition to

z E-mail: joanna.oommen@kaust.edu.sa; muhammad.hussain@kaust.edu.sa understanding the temperature-wise performance of NIMs and determining the types of bonding within the NIM system. We acquired the ${ }^{13} \mathrm{C}$ and ${ }^{29} \mathrm{Si}$ spectra of different NIM compounds using MAS and CP NMR spectroscopy. The NIM samples used in our experiments consisted of a $\mathrm{SiO}_{2}$ nanoparticle core attached to a sulfonate group through a silane spacer (inset of Fig. 1). The corona was composed of poly(ethylene glycol) (PEG) and polystyrene (PS) of different molecular weights. We used three samples, A, B, and C, in our experiments. Sample A has a $9000 \mathrm{Mw}$ PEG corona, B has a 2000 Mw PEG corona, and C has 6500 Mw PS corona.

The chemical shift in an NMR spectrum describes the dependence of the NMR on the electronic environment around the nucleus; it has thus been used as a kind of fingerprint for identifying the molecular composition of a sample.

High resolution ${ }^{13} \mathrm{C}$ and ${ }^{29} \mathrm{Si}$ MAS NMR spectra were collected at 100.6 and $79.5 \mathrm{MHz}$, respectively, on an AVANAC III 400 Bruker instrument. The ${ }^{29} \mathrm{Si}$ NMR spectra were recorded at temperatures ranging between 300 and $383 \mathrm{~K}$ using a Bruker BCU-Xtreme temperature control system.

Figure 1 shows the Si spectra obtained from the three samples. The first observation is that the ${ }^{29} \mathrm{Si}$ NMR spectra demonstrate similar chemical shifts at all temperatures, verifying the similarity of the chemical environment around the $\mathrm{SiO}_{2}$ core and the stability of NIMs in relation to temperature. Both hydrogen and covalent bonds could be formed to connect the organic polymer with the $\mathrm{SiO}_{2}$ core. ${ }^{10,11}$ Hydrogen bonds could be formed between the basic group of the hydrogen acceptor in the polymer and the oxygen atom of $\mathrm{SiO}_{2}$. Because the chemical shift of $\mathrm{SiO}_{2}$ did not show any change with temperature elevation, these results suggest the presence of a stable, covalent bond between the $\mathrm{SiO}_{2}$ core and the sulfonate group through the silane spacer.

Second, we observed two peaks in all the ${ }^{29} \mathrm{Si}$ spectra, the smaller of the peaks being very prominent in sample B. Thus, we detect two types of $\mathrm{Si}$ : One is $\mathrm{Si}$ in the $\mathrm{SiO}_{2}$ core indicated by the bigger peak, and the second is Si present in the spacer material. The latter has a smaller quantity in any given sample as it is only bonded to the nanoparticles on the periphery of the $\mathrm{SiO}_{2}$ core (see inset of Fig. 1). In this context, it is important to make two comparisons: one between the two samples with PEG corona (samples A and B) and the other between samples with PEG and PS corona (samples A and B and sample C, respectively). Sample A contains a 9000 Mw PEG corona, whereas B contains a 2000 Mw PEG corona. Therefore, we expect a larger core, hence, a greater difference between the amounts of the $\mathrm{SiO}_{2}$ core and the $\mathrm{Si}$ spacer in A. Correspondingly, we see a very small peak adjacent to the major Si peak in A (and C), whereas we see a much stronger and sharper peak in B.

The next observation from the ${ }^{29} \mathrm{Si}$ spectra is the shape of the 


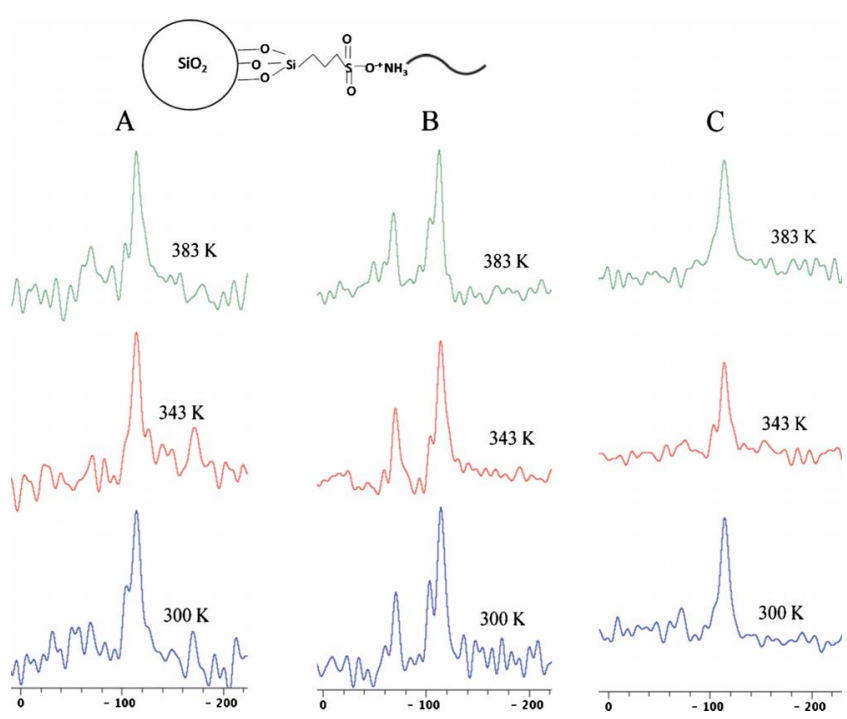

Figure 1. (Color online) Effect of temperature variation on NIM compounds: A (PEG Mw: 9000), B (PEG Mw: 2000), and C (PS Mw: 6500). Inset: Proposed structure for NIMs with core, corona, and counterions.

major peaks and how these shapes change with temperature. Although we observe a notch mostly on the left half of the peaks of the three samples, in sample A, the notch shifted to the right half when the temperature increased from 300 to $343 \mathrm{~K}$ and then it shifted back to the left before $383 \mathrm{~K}$. In sample B, the notch is sharper at lower temperatures. Sample $\mathrm{C}$ has a mostly clean peak, but at $\sim 343 \mathrm{~K}$ a small notch appears and seems to shave off some of the height from the original peak. The notch disappears upon increasing the temperature to $383 \mathrm{~K}$. We performed the experiment in reverse by decreasing the temperature and concluded that these changes in the shapes of the peaks were irreversible. A possible explanation for this is the presence of small amounts of leftover solvent, which evaporates on heating, leading to the observed changes in the spectrum. Another possibility is the dynamics of the $\mathrm{SiO}_{2}$ core and the silane spacer coupling the core with its corona, suggesting some sort of internal rearrangement of $\mathrm{Si}$.

We used CP MAS to study the polymeric corona attached to the $\mathrm{SiO}_{2}$ core. The ${ }^{13} \mathrm{C}$ spectrum is used to differentiate between different aliphatic and aromatic polymers and to quantify different components within the same polymer. Figure 2 shows the ${ }^{13} \mathrm{C}$ CP MAS spectrum of sample $\mathrm{C}$ where the integrations of the three main peaks

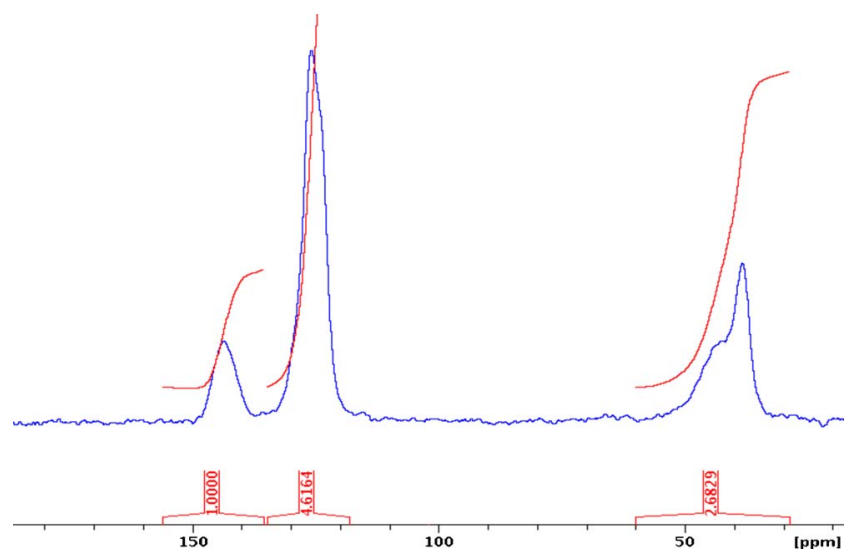

Figure 2. (Color online) ${ }^{13} \mathrm{C}$ spectrum for sample $\mathrm{C}$ clearly shows a major aromatic PS component.

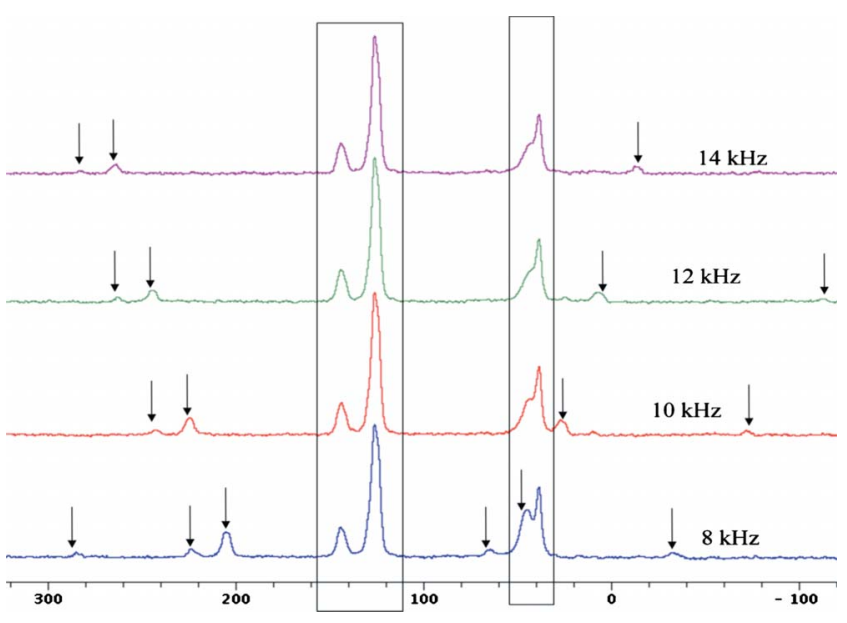

Figure 3. (Color online) ${ }^{13} \mathrm{C}$ NMR spectra of sample $\mathrm{C}$ at different spinning rates. This figure shows the effect of the spinning rate on the position of the artificial side band lines.

are 1.00 and 4.61 in the aromatic region and 2.68 in the aliphatic region, which correctly indicates the major aromatic PS component. The two peaks represent quaternary carbon at $150 \mathrm{ppm}$ and alkene carbon at $125 \mathrm{ppm}$. We resolved the problem of artificial side band lines associated with MAS spectra by gathering ${ }^{13} \mathrm{C}$ spectra at different spinning rates to distinguish between real peaks and side band lines (Fig. 3). On varying spinning rates from 8 to $14 \mathrm{kHz}$, the artificial side band lines were observed to shift in position (marked by arrows), whereas the real peaks remained fixed (within box). Once the real peaks are identified, we determine aromatic and aliphatic component ratios from the peak heights.

Our solid-state NMR and CP MAS NMR experiments have led to new insights about the behavior of NIMs in relation to temperature and confirmed existing hypotheses about the structure and composition of NIMs. These initial experiments are important additions to the existing body of knowledge on NIMs as we can now explore high temperature applications for NIMs. We hope to extend our study with NMR-diffusion experiments on NIMs soon.

\section{Acknowledgments}

The authors acknowledge the sample preparation and NMR experimental analysis performed at Cornell University and KAUST NMR Core Laboratories, respectively.

King Abdullah University of Science and Technology assisted in meeting the publication costs of this article.

\section{References}

1. A. B. Bourlinos, R. Herrera, N. Chalkias, D. D. Jiang, Q. Zhang, L. A. Archer, and E. P. Giannelis, Adv. Mater, 17, 234 (2005).

2. P. Agarwal, H. B. Qi, and L. A. Archer, Nano Lett., 10, 111 (2010).

3. R. Rodriguez, R. Herrera, L. A. Archer, and E. P. Giannelis, Adv. Mater, 20, 4353 (2008).

4. A. B. Bourlinos, A. Stassinopoulos, D. Anglos, R. Herrera, S. H. Anastasiadis, D. Petridis, and E. P. Giannelis, Small, 2, 513 (2006).

5. S. C. Warren, M. J. Banholzer, L. S. Slaughter, E. P. Giannelis, F. J. DiSalvo, and U. B. Wiesner, J. Am. Chem. Soc., 128, 12074 (2006).

6. A. B. Bourlinos, E. P. Giannelis, Q. Zhang, L. A. Archer, G. Floudas, and G. Fytas, Eur. Phys. J. E, 20, 109 (2006)

7. B. H. Han, M. A. Winnik, A. B. Bourlinos, and E. P. Giannelis, Chem. Mater, 17, 4001 (2005).

8. G. Qi, K. A. Lin, Y. Park, E. P. Giannelis, and A. Park, in AIChE Annual Meeting (2009), http://aiche.confex.com/aiche/2009/webprogram/Paper 166988.html, last accessed Aug 13, 2010.

9. B. Smarsly and H. Kaper, Angew. Chem., Int. Ed., 44, 3809 (2005).

10. Y. Wei, D. Jin, C. Yang, M. C. Kels, and K. Y. Qiu, Mater. Sci. Eng., C, 6, 91 (1998).

11. S.-I. Han, J. S. Lim, D. K. Kim, M. N. Kim, and S. S. Im, Polym. Degrad. Stab., 93, 889 (2008). 